\title{
Article \\ Effect of the Fibre Orientation Distribution on the Mechanical and Preforming Behaviour of Nonwoven Preform Made of Recycled Carbon Fibres
}

\author{
Jean Ivars, Ahmad Rashed Labanieh *(D) and Damien Soulat $(\mathbb{D}$ \\ GEMTEX Laboratory, ENSAIT, University Lille North of France, F-59100 Roubaix, France; \\ jean.ivars@ensait.fr (J.I.); damien.soulat@ensait.fr (D.S.) \\ * Correspondence: ahmad.labanieh@ensait.fr
}

check for

updates

Citation: Ivars, J.; Labanieh, A.R.; Soulat, D. Effect of the Fibre Orientation Distribution on the Mechanical and Preforming Behaviour of Nonwoven Preform Made of Recycled Carbon Fibres. Fibers 2021, 9, 82. https://doi.org/ 10.3390/fib9120082

Academic Editors: Ahmad Rashed Labanieh and Vincenzo Fiore

Received: 18 October 2021

Accepted: 1 December 2021

Published: 8 December 2021

Publisher's Note: MDPI stays neutral with regard to jurisdictional claims in published maps and institutional affiliations.

Copyright: (c) 2021 by the authors. Licensee MDPI, Basel, Switzerland. This article is an open access article distributed under the terms and conditions of the Creative Commons Attribution (CC BY) license (https:/ / creativecommons.org/licenses/by/ $4.0 /)$.

\begin{abstract}
Recycling carbon-fibre-reinforced plastic (CFRP) and recovering high-cost carbon fibre (CF) is a preoccupation of scientific and industrial committees due to the environmental and economic concerns. A commercialised nonwoven mat, made of recycled carbon fibre and manufactured using carding and needle-punching technology, can promote second-life opportunities for carbon fibre. This paper aims to evaluate the mechanical and preforming behaviour of this nonwoven material. We focus on the influence that the fibre orientation distribution in the nonwoven material has on its mechanical and preforming behaviour at the preform scale, as well as the tensile properties at composite scale. The anisotropy index induced by fibre orientation is evaluated by analysing SEM micrographs using the fast Fourier transform (FFT) method. Then, the anisotropy in the tensile, bending, and preforming behaviour of the preform is inspected, as well as in the tensile behaviour of the composite. Additionally, we evaluate the impact of the stacking order of multi-layers of the nonwoven material, associated with its preferred fibre orientation (nonwoven anisotropy), on its compaction behaviour. The nonwoven anisotropy, in terms of fibre orientation, induces a strong effect on the preform mechanical and preforming behaviour, as well as the tensile behaviour of the composite. The tensile behaviour of the nonwoven material is governed by the inter-fibre cohesion, which depends on the fibre orientation. The low inter-fibre cohesion, which characterises this nonwoven material, leads to poor resistance to tearing. This type of defect rapidly occurs during preforming, even at too-low membrane tension. Otherwise, the increase in nonwoven layer numbers leads to a decrease in the impact of the nonwoven anisotropy behaviour under compaction load.
\end{abstract}

Keywords: recycled carbon fibre; nonwoven preform; mechanic of fibrous reinforcement; preforming

\section{Introduction}

In 2017, the global demand for carbon fibres, to be used in 114.7 ktons of carbon-fibrereinforced plastics (CFRP), was approximately 70.5 ktons [1], and it could reach 122.8 ktons in 2022 [2]. These CFRPs are used mainly in industrial applications, such as aerospace, defence, automotive, wind energy, sports equipment, and construction. In accordance with this higher demand, it is estimated that as much as $30 \%$ of the total material used in the manufacturing of carbon fibre parts is wasted, primarily in the form of scrap prepreg [3]. To reuse carbon fibres from CFRP wastes, mechanical and fibre reclamation technologies (pyrolysis and solvolysis) are first employed for recycling, and are intensively described in the literature $[4,5]$. Due to their vital role in the composite performance, the characteristics of the recycled carbon fibres ( $\mathrm{rCF}$ ) obtained from these recycling technologies are identified in terms of their fibre length, tensile strength, purity, and sizing. In a recent review, Kurshid et al. [6] classified the fibre length into three main categories (short: $<6 \mathrm{~mm}$, medium: 6-25 mm, and long: 25-300 mm) depending of the reclamation technologies and type of wastes. For short fibres, the recycled fibres are currently used in relatively low-performance applications such as injection moulding and 3D printing; however, other 
processes, such as carding or wet deposition, which are used to ensure that nonwoven fabrics retain their fibre length, offer improved mechanical properties that could allow recycled fibre composites to be used in higher-performance applications [7]. The main advantages of the nonwoven process are an excellent economic efficiency due to their high production output, high flexibility regarding produced nonwoven properties (areal weight and degree of compaction), ability to modify the line to minimize fibre damage, and compact carding process possibilities, which combines several conversion processes to produce a homogeneous textile surface from a fibre blend. Many studies have looked at the use of this technology for $\mathrm{rCF}[8,9]$. Akonda et al. [10] used staple carbon fibres, which were mixed with staple PET fibres to produce continuous slivers using a modified carding process, and then composite samples by hot-pressing. Hengstermann et al. [11] produced two kinds of hybrid yarn from, respectively, virgin carbon fibres and $\mathrm{rCF}$, which were mixed with PA6 fibres, by using a laboratory, long staple carding machine. UD composite plates were then manufactured by hot pressing. Some works used manufacturing methods to mix recycled carbon fibres with polymer fibres to produce preforms, which are then stacked in a mould and compression moulded. Wei et al. [12] described a wet-laid process, used to mix rCF and PA6 fibres together in a mat of randomly oriented fibres, which were formed in composite samples by compression moulding. Barnett et al. [7] compared the mechanical properties of composite samples obtained by compression moulding with PPS film and repurposed virgin carbon fibre nonwovens manufactured by the wet-laid process and recycled carbon fibre nonwovens provided by ELG carbon fibre. In these studies, the distribution of the length and orientation of fibres in a nonwoven mat were particularly studied for their critical influence on the mechanical properties at composites scales. Manis et al. [8] analysed the influence of the web formation in the carding machine by measuring the fibre length at four positions along the carding machine. The nonwoven fabrics have no directionality and are randomly distributed in the longitudinal and transverse direction [12]. Their anisotropy was studied by many authors, especially those using image analysis to quantify the fibre orientation distribution $[13,14]$. From the images obtained by scanning electronic microscopy (SEM) and analysed by computing the histogram of pixels' preferred orientations using grey-level granulometry, Gager et al. [15] presented a new method to analyse the fibre orientation distribution (FOD) of the flax nonwovens. Yu et al. [16] used image analysis by Hough transform to investigate the fibres' orientation distribution within needle-punched nonwovens. In a study where waste carbon fibres and polyamide fibres were processed on a double-cylinder card machine, Kurshid et al. [17] followed the fibre orientation in the function of different technological parameters of the carding process. Jlassi et al. [18] compared the tensile properties in the machine manufacturing direction (MD) and cross direction (CD) of composites made by compression moulding from carbon-carded, and pre-needled, nonwoven, and PA6 films, but no index of ratio of the anisotropy index is defined. On recycled carbon fibre nonwovens, Barnett et al. [7] carried out dry-scale tensile tests (before consolidation) to give an indication of their degree of anisotropy, but they focused more on the mode of failure of their specimens than on defining an anisotropy index from their experimental curves.

In this study, we aim to analyse the properties of rCF nonwovens obtained by carding, cross-lapping, and needle-punching at different scales: fibre, preform, and composite. The characterisation methods are described for each scale. Then, the results are presented, followed by a discussion. We focus on the influence of the anisotropy of the rCF nonwoven associated with the fibre orientation distribution on the mechanical and preforming behaviour of the preform and the mechanical performance of the composite.

\section{Materials}

In the present study, the mechanical and preforming behaviour of a nonwoven preform provided by ELG is evaluated. The nonwoven is made from $100 \%$ recycled carbon fibres (rCF), classified as a standard modulus fibre SM450 with a strength of 4-5 GPa, as reported in the product's datasheet. The nonwoven is manufactured by carding cross-lapping and 
needle-punching technology $[7,8]$. The manufacturing direction of the nonwoven (MD) along the needle-punching machine corresponds to the perpendicular direction of the card line production, which corresponds to the cross direction of the nonwoven (CD).

The nonwoven fabric is characterised by an areal density of $193\left( \pm 7.15 \mathrm{~g} / \mathrm{m}^{2}\right)$ and a thickness of $3.85( \pm 0.17 \mathrm{~mm})$, measured under a pressure load of $0.1 \mathrm{KPa}$. These identifications are performed, following NF EN 12127 standard [19] for the areal density, and the standard NF EN ISO 5084 [20] for the thickness. The tests are conducted on ten specimens prepared in both nonwoven main directions: the manufacturing direction (MD) and cross direction (CD). The low values, obtained for the standard deviation of the measured areal density and thickness, indicate a homogeneous material distribution and homogeneous condensation, obtained by needle-punching across the nonwoven surface.

\section{Methods}

The physical and mechanical properties of the rCF nonwovens are characterised at three scales: fibre scale, preform scale, and composite scale. This allows for an understanding of the interaction between the different scales, and an analysis of the influence of the fibre orientation distribution on the mechanical behaviour of the nonwoven preform and manufactured composite. The methods used for this experimental approach are described at each scale.

\subsection{Fibre Scale}

The geometry of the recycled carbon fibre is characterised in terms of length and diameter by carefully extracting and separating the individual fibres from the nonwoven. To measure the fibre length, they are laid down on a paper and then a picture is taken and analysed using image analysis with the software ImageJ [21]. To measure the diameter of the fibre, an optical microscope is used, with $\times 40$ magnification and $0.42 \mu \mathrm{m}$ resolution. The fibres are extracted from five different nonwoven zones. Diameter and length measurements are performed on 50 fibres.

\subsection{Preform Scale}

\subsubsection{Fibre Orientation}

Identification of the orientation distribution of the fibre network, weighted by the fibre content at each angle, is essential to identify the anisotropy of the nonwoven and, consequently, the composite. The fibre orientation distribution can be identified from the captured SEM micrographs by the developed image analysis technique using an imagebased fibre orientation and alignment calculator function, developed by Barocas [22]. This function is based on the fast Fourier transform (FFT) method [22-24], which provides better accuracy than the mean intercept length method (MIL) and line fraction deviation method (LFD) $[23,24]$.

SEM micrographs are captured for samples picked up from different zones in the nonwoven. Then, they are analysed by a developed MATLAB code in association with the Barocas function [22]. This consists of converting the captured binary grey-scale image into a frequency space expressed in polar coordinate. Then, the normalized orientation distribution function is defined from the number of points on the radius line at each angle and their intensity value. This reveals the number of fibres at each angle. Then, the orientation tensor $(\mathrm{F})$ is defined according to the equations described in [24].

The eigenvalues $\left(\lambda_{1}, \lambda_{2}\right)$ and eigenvectors of the obtained orientation tensor $(F)$ are determined to identify the anisotropy index $\alpha$ according to the following equation, Equation (1):

$$
\alpha=1-\frac{\lambda_{1}}{\lambda_{2}}
$$

An isotropic network has an index value equal to zero, whereas a unidirectional network has an index value equal to one. The anisotropy index gives the preferred orientation of the fibres in the nonwoven, resulting from the manufacturing process (carding, 
cross-lapping, and needle-punching). This anisotropy index is compared with those computed from the mechanical properties identified at the scales of the nonwoven and the manufactured composite.

An example of this conducted procedure can be illustrated on a captured SEM at 1000 magnification, as in Figure 1a. The image is trimmed into a square binary image, Figure $1 \mathrm{~b}$, then converted into a frequency space with a polar coordinate, as in Figure 1c. The eigenvectors of the length-weighted fibre orientation tensor are drawn on the treated image, Figure 1d, with a polar representation of the fibre orientation distribution function, as in Figure 1e. The identified anisotropy index $(\alpha)$ for the given micrographs is 0.98 .

a)

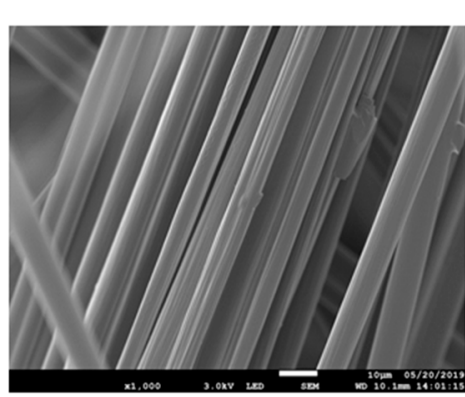

b)

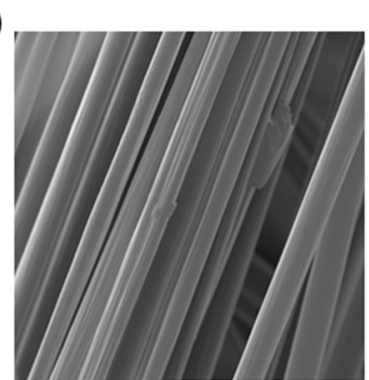

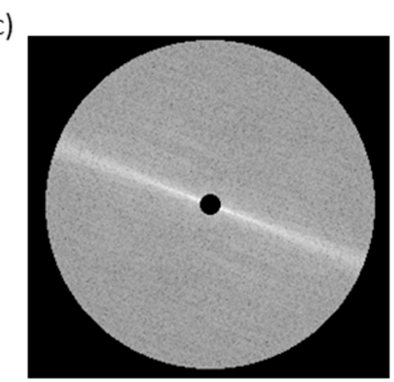

$90 \quad 20$ d) ,

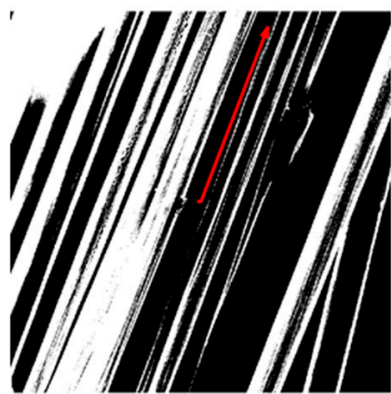

e)

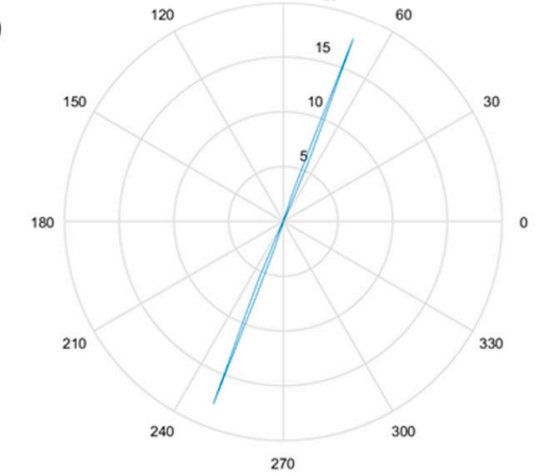

Figure 1. Example of performed analysis on SEM micrograph captured at 2000 magnification (a) by FFT method, trimming into square image (b), converting into frequency space with polar coordinate (c), indication eigenvectors (in red) (d), and polar fibre orientation distribution (e).

\subsubsection{Compaction Test}

If the compaction behaviour of textiles is well documented for different reinforcement architectures, such as woven, NCF, etc. [25-30], few studies have been conducted on nonwovens. The compaction behaviour of the rCF nonwoven multilayers is identified in the present study. This is helpful in the manufacture of the nonwoven composite, using a compression moulding process and analysing the evolution of the fibre volume fraction of the nonwoven with the applied pressure. Furthermore, the influence of the preferred fibre orientation on the compressibility of a multilayer of the nonwoven can be evaluated by changing the stacking order of the layers. The test is performed on an MTS Criterion tensile machine with a load cell of $250 \mathrm{kN}$ at a crosshead displacement rate of $1 \mathrm{~mm} / \mathrm{min}$. The samples are compacted between two metallic plates until a pressure of 60 bar. By considering the capacity of the load cell and sample dimension, the pressure measurement is performed, with an accuracy of 0.39 bar. The thickness of the specimen is measured by the crosshead displacement reading after subtracting the compliance of the tensile machine (denoted C in Equation (2)), as mentioned in [25-30]. All samples are tested on the same machine, using the same thickness measurement technique, which can establish a comparison between them. Stacks of four and eight layers (with a size of $80 \mathrm{~mm} \times 80 \mathrm{~mm}$ for each layer) are prepared with two stacking orders. In the first configuration, all the layers are stacked in the same orientation, denoted MD/MD. In the second group, the 
layers are stacked in cross-ply configuration. By considering the MD direction for the nonwoven layer, each layer is placed orthogonal to the preceding one. In this way, the orientation of the successive layers is alternated between the MD and CD directions. The stacking order is denoted as (MD/CD). For each test, the fibre volume fraction $\left(v_{f}\right)$ of the multilayer specimen is calculated as a function of the crosshead displacement, with the pressure increasing according to the following equation, Equation (2):

$$
v_{f}=\frac{A_{w} N}{\rho_{f}\left(h_{0}-d+F C\right)}
$$

with $A_{w}$ as the areal density of one layer $\left(\mathrm{g} / \mathrm{m}^{2}\right), N$ the number of stacked layers, $\rho_{f}$ is the density of fibre $\left(\mathrm{g} / \mathrm{m}^{3}\right), h_{0}$ the initial cavity height $(\mathrm{m}), d$ the crosshead displacement $(\mathrm{m}), F$ is the machine load $(N)$, and $C$ is the machine compliance $(\mathrm{m} / N)$. The evolution of the fibre volume fraction and of the thickness per layer with the pressure is analysed, as described in [26-28].

\subsubsection{Tensile Test}

Tensile tests on nonwoven samples, before impregnation, are conducted according to the NF EN 9073 standard [31] on a Criterion MTS tensile machine, with a load cell of $1 \mathrm{kN}$ capacity. The test speed is set to $100 \mathrm{~mm} / \mathrm{min}$ and the specimen is prepared with a gauge length of $200 \mathrm{~mm}$ and a width of $50 \mathrm{~mm}$. The tensile test is performed on specimens in the nonwoven manufacturing direction (MD), in the cross direction (CD), and in the bias direction ( $45^{\circ}$ relative to the manufacturing direction). Anisotropy in the tensile behaviour of the nonwoven is determined by the ratio of the maximal loads, as well as by the ratio of the tangent modulus between the two main directions: MD and CD.

\subsubsection{Bending Test}

The flexural rigidity of the nonwoven is identified using a cantilever apparatus according to the NF EN ISO 9073-7 standard [32]. The test is conducted in the two main directions (MD and CD). Six specimens are tested in each direction, with $250 \mathrm{~mm}$ length and $25 \mathrm{~mm}$ width. The flexural rigidity per unit width $\mathrm{G}\left(\mathrm{N} \cdot \mathrm{m}^{2} / \mathrm{m}\right)$ is calculated from the measured bending length $\mathrm{L}(\mathrm{m})$, considering the areal density $A_{w}\left(\mathrm{~g} / \mathrm{m}^{2}\right)$ and the acceleration due to gravity $\mathrm{g}\left(\mathrm{m} / \mathrm{s}^{2}\right)$, according to Equation (3). Anisotropy in the bending behaviour is estimated by the ratio of flexural rigidity in the two main directions: $\mathrm{MD}$ and $\mathrm{CD}$.

$$
G=\frac{g A_{w} L^{3}}{8}
$$

\subsubsection{Preforming}

The drapability behaviour of the $\mathrm{rCF}$ nonwoven is performed on a preforming device developed in the GEMTEX laboratory [33-35]. On this device, a $250 \times 250 \mathrm{~mm}^{2}$ nonwoven sample, placed between the die and the blank-holder plates, is preformed by a $150 \mathrm{~mm}$ diameter half-spherical punch, as shown in Figure 2.

The punch movement is controlled by a pneumatic actuator with a constant mounting speed $(10 \mathrm{~cm} / \mathrm{s})$. A load cell is placed between the punch and actuator to record the preforming force. Both die and blank holder plates are thick enough to ensure a homogeneous pressure on the specimen at the contact area with the two plates. The test is performed under a low blank holder pressure of $0.017 \mathrm{MPa}$. The blank holder plates are transparent to track the specimen edges' displacement after preforming via a digital camera placed on the top side of the blank holder plate. The displacement of the specimen edges relative to their initial position is denoted as draw-in distance. These distances are measured thanks to pictures captured before (Figure $3 a$ ) and at the end of the preforming process (Figure 3b) using ImageJ software [19] for the image analysis. Draw-in distances, illustrated in Figure 3b, depend on the applied blank holder pressure and specimen orientation. Furthermore, white dots are speckled on the sample surface before preforming along the 
$x$-axis, $y$-axis, and both diagonals, at $45^{\circ}$ and $-45^{\circ}$. They are made with a constant radial interval of $2 \mathrm{~cm}$ relative to the hole centre in the blank-holder plate. These dots mean that the deformation anisotropy of the sample can be followed after preforming by measuring the eccentricity index of the deformed initial circle, created from white dots, as in Figure $3 c$, into an ellipse. Omrani et al. [33] used this method of tracking markers on nonwoven flax to analyse deformation evolution inside the nonwoven material during this preforming step, but without association with an anisotropy ratio.

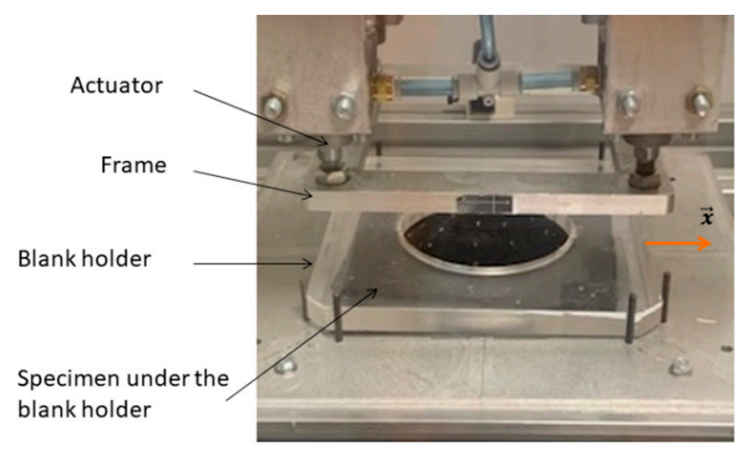

Figure 2. Preforming machine.

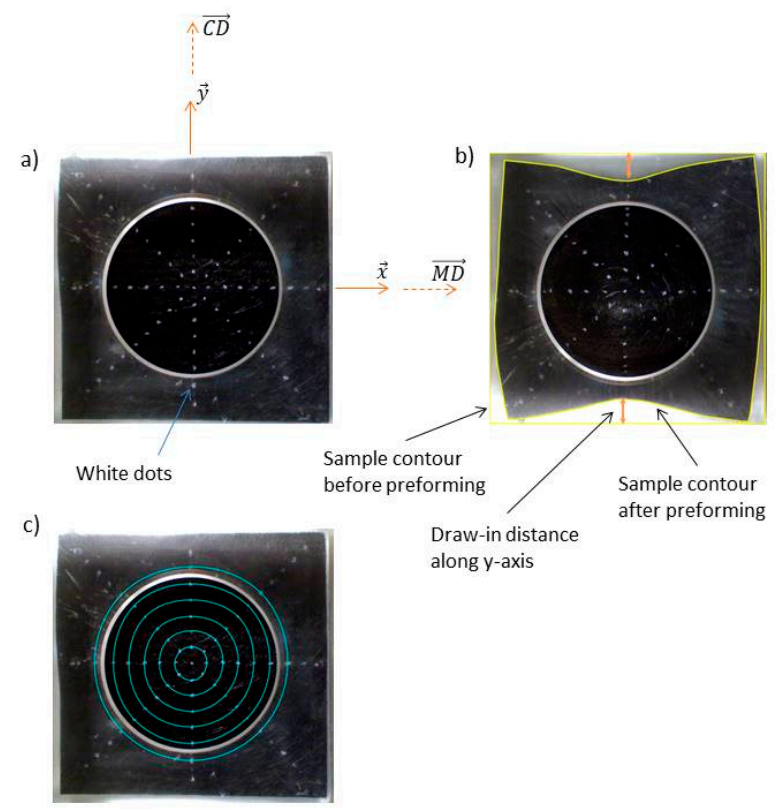

Figure 3. Specimen before preforming placed under blank-holder plate (a), formed preform under the blank-holder plate at the end of the punch course (b), and initial state of the concentric circles created from white dots marked on the specimen (c).

\subsection{Composite Scale}

The thermocompression moulding technique is used to manufacture composite samples from rCf nonwoven layers with polypropylene plastic (PP). Four nonwoven layers are stacked in the same orientation, alternating with three PP film layers. The last is made from PP grain by thermos-compressing moulding with a $0.5 \mathrm{~mm}$ thickness. The stacked layers are heated under a 4 bar pressure until reaching $220^{\circ} \mathrm{C}$; then, a 9.55 bar is applied for 1 min before cooling with air under the maintained pressure. At this stage, the laminates are manufactured without applying any sizing agent. The fibre and porosity content $\left(v_{f}\right.$ and $v_{p}$ ) in each manufactured laminate are estimated according to the method described 
by Berges et al. [36]. This method is based on the areal density $\left(A_{r}\right)$ of the preform, number of lamina $(N)$ and fibre density $\left(\rho_{f}\right)$ in a tested sample, as detailed in Equation (4):

$$
w_{f}=A_{r} N S / m, \rho_{c}=m /(h S), v_{f}=w_{f} \cdot \rho_{c} / \rho_{f}, v_{p}=1-v_{f}-\left(1-w_{f}\right) \rho_{c} / \rho_{m}
$$

where $w_{f}$ is the mass fraction of fibre and $\rho_{c}$ and $\rho_{m}$ are the density of the tested composite specimen and the matrix, respectively, with masse $m$, surface area $S$, and thickness $h$.

The tensile properties of the manufactured laminates are characterised according to ASTM D3039 standard [37] using tensile test machine MTS Criterion, equipped with a load cell with a capacity of $250 \mathrm{kN}$. The tests are conducted with a gauge length of $150 \mathrm{~mm}$, a width of $15 \mathrm{~mm}$, and a crosshead displacement rate of $1 \mathrm{~mm} / \mathrm{s}$. Tensile tests are performed in both $\mathrm{MD}$ and $\mathrm{CD}$ directions, respectively, of the $\mathrm{rCF}$ nonwoven. The tensile modulus is computed on the obtained stress-strain curve in the strain range of (0.1-0.3). The strength and strain failure are also reported. The anisotropy index of the tensile composite behaviour is computed from the ratio of the tensile strength and modulus in both directions.

\section{Results and Discussion}

\subsection{Recycled Carbon Fibres Properties}

The box plot of the measured values of the fibre length is given in Figure 4a, with an average value of $72.17 \mathrm{~mm}$ and a standard deviation of $9.9 \mathrm{~mm}$. The measured fibre diameter is given in a box plot on Figure $4 \mathrm{~b}$, with an average value of $9.01 \mu \mathrm{m}$ and standard deviation of $0.68 \mu \mathrm{m}$.

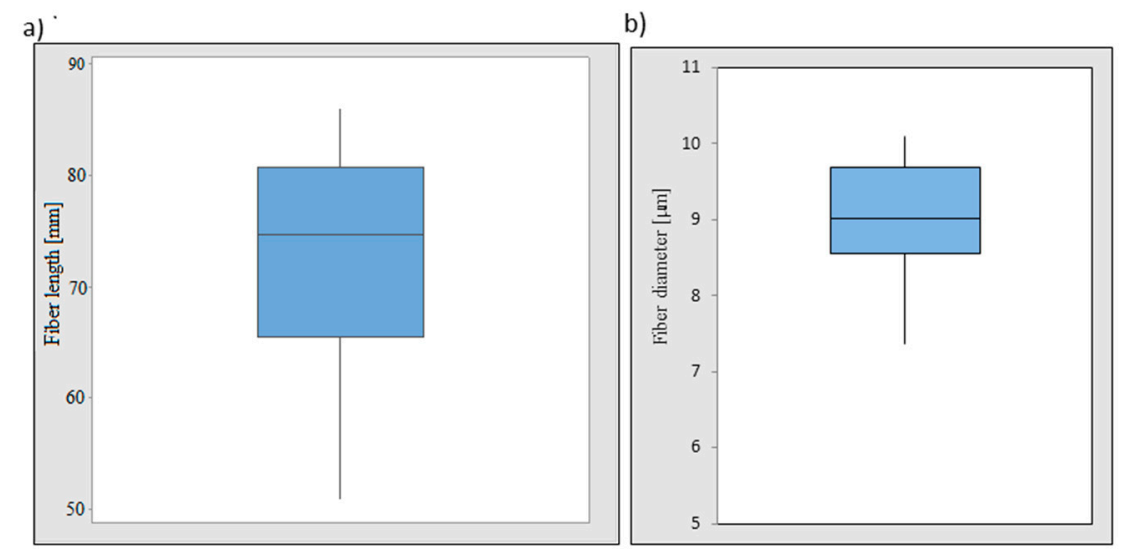

Figure 4. Box plot of the measured length (a) and the diameter (b) of extracted recycled carbon fibre.

\subsection{Nonwoven}

\subsubsection{Fibre Orientation}

The fibre orientation distribution analysis is conducted on 48 micrographs, captured at two magnification levels $(\times 100$ and $\times 50)$, Figure $5 a, d$, respectively. They are made on samples that are randomly picked on the nonwoven material, without referring to the image orientation relative to the nonwoven main axis. The anisotropy index of the nonwoven is computed for the two micrograph groups by Equation (1), after determining the characterising eigenvectors and eigenvalues by means of the FFT image analysing system. One example of the performed analysis is presented in Figure 5b,e, with sketched eigenvectors for the fibre orientation distribution on the analysed micrograph in red arrows. The polar plot of the fibre orientation distribution is also obtained by this analysis, as in Figure $5 c, f$. An obvious accordance is observed between eigenvector orientations, Figure $5 b$, and fibre orientation distribution, Figure $5 \mathrm{c}$,f. The box plot of the anisotropy index for the two micrograph groups, with a magnification level of $\times 50$ and $\times 100$, is presented in Figure 6, with a mean of 0.39 and 0.4 and a standard deviation value of 0.15 and 0.13 , respectively. Less scatter is noted on the micrographs captured at $\times 100$ magnification. The 
values obtained for anisotropic index highlight the preferred orientation for fibres in the nonwoven network.
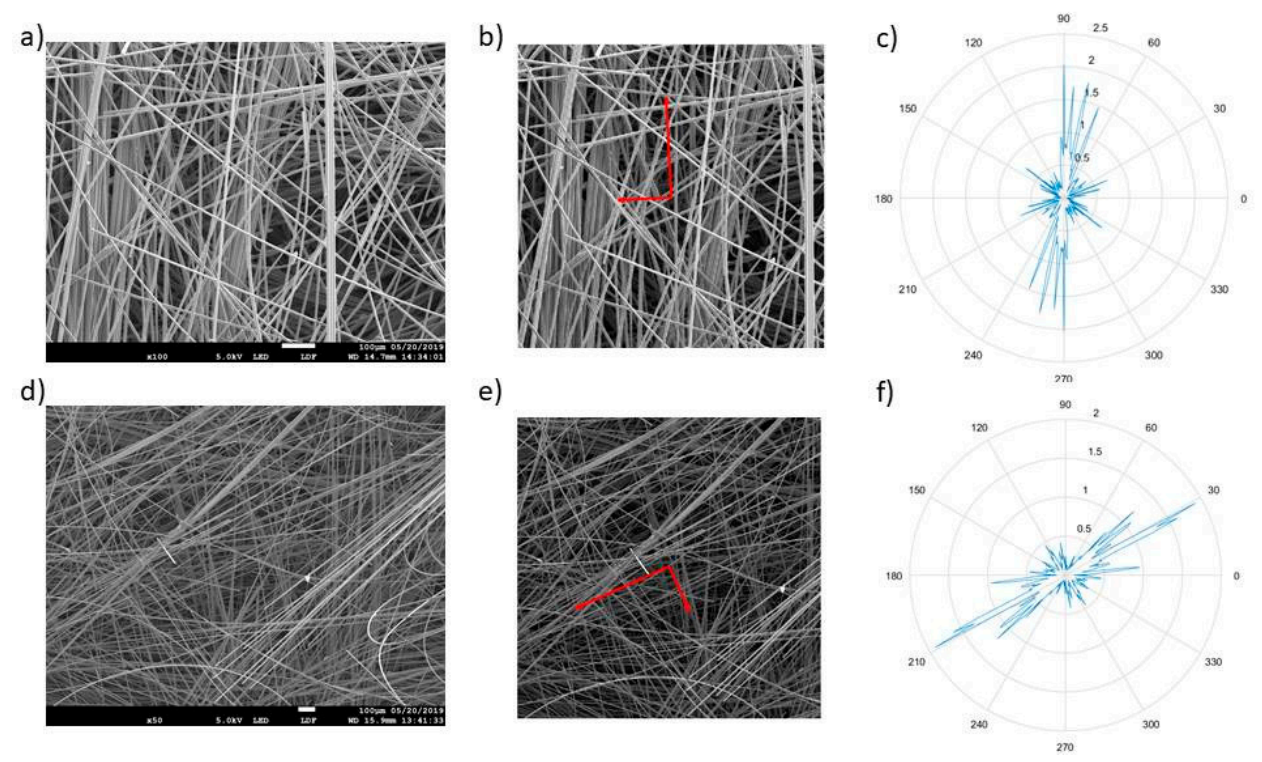

Figure 5. Example of the captured SEM micrograph at $\times 100$ and $\times 50$ magnification $(\mathbf{a}, \mathbf{d}$, respectively), analysed image with two sketched eigenvectors $(\mathbf{b}, \mathbf{e})$, and polar plot distribution of the fibre orientation $(\mathbf{c}, \mathbf{f})$ at both magnifications, $\times 100$ and $\times 50$, respectively.

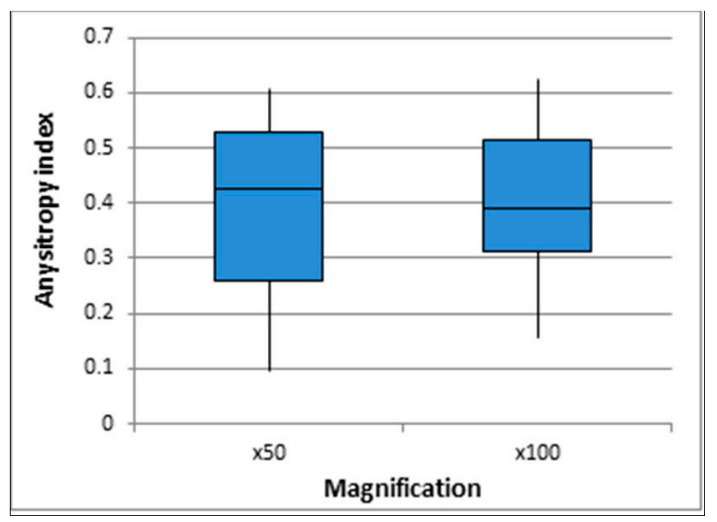

Figure 6. Anisotropy index estimated for SEM micrographs captured with $\times 50$ and $\times 100$ magnification.

\subsubsection{Compaction Behaviour of rCF Nonwoven Material}

The compaction test is performed on four and eight nonwoven layers, stacked in two orientation configurations (MD/MD and MD/CD). The estimated fibre volume fraction of the specimens is computed based on Equation (2) as a function of the tracked displacement of the machine crosshead after compliance subtraction. The pressure-fibre volume fraction curve, Figure 7a, is characterised with three distinctive phases [26]. At the beginning and end of the curve, a linear relationship is observed between the pressure and the fibre volume fraction. However, at the transition phase between these two, this relationship is nonlinear. First, the volume fraction of the fibres increases considerably with a small increase in pressure because of the reorganisation of the fibres within the nonwoven to fill the inter-fibres gaps. Then, by reducing the inter-fibre gaps, the fibres bend, slide, and nest between other fibres from the same layer and other layers. This requires a higher pressure, which exponentially increases in the second phase. Finally, in the third phase, laterally compressing the fibres strongly increases the pressure, with a slight increase in the fibre volume fraction. Extending the second phase before fibre compression indicates the compressibility of the structure. The specimens composed of eight layers showed a wide 
second phase in comparison with the specimens of four layers. This results in a higher fibre volume fraction in the eight-layer specimen in comparison with the four-layer specimen at the same applied pressure. The normalised thickness per layer relative to its thickness at 0.01 bar in the two configurations (four and eight layers) is estimated by considering the specimen thickness relative to the number of constitutive layers. The thickness per layer in the eight-layer specimen is highly lower than that in the four-layer specimen. It can signify that the fibres' inter-layer nesting is more significant with an increase in the number of layers. The stacking order of samples with the two orientation configurations (MD/MD and $\mathrm{MD} / \mathrm{CD}$ ) has an impact in the compaction behaviour of the nonwoven material in the case of four layers. A higher pressure is required for the same stacking order (MD/MD) in comparison with the alternate orientation $(\mathrm{MD} / \mathrm{CD})$ to attain the same fibre volume fraction. As an example, a fibre volume fraction of $25 \%$ and a pressure of 39 bar is required for four layers in the MD/MD stacking order, while a pressure of 27 bar is required for the MD/CD stacking order, as in Figure 7a. Similarly, a layer in a four-layer nonwoven with an MD/MD configuration will have a higher thickness in comparison with an MD/CD configuration at the same applied pressure, as in Figure $7 \mathrm{~b}$. However, this impact is low in the case of eight layers. Furthermore, the impact of the number of layers is more significant than the stacking order and, consequently, an anisotropy index could not be defined from these tests.
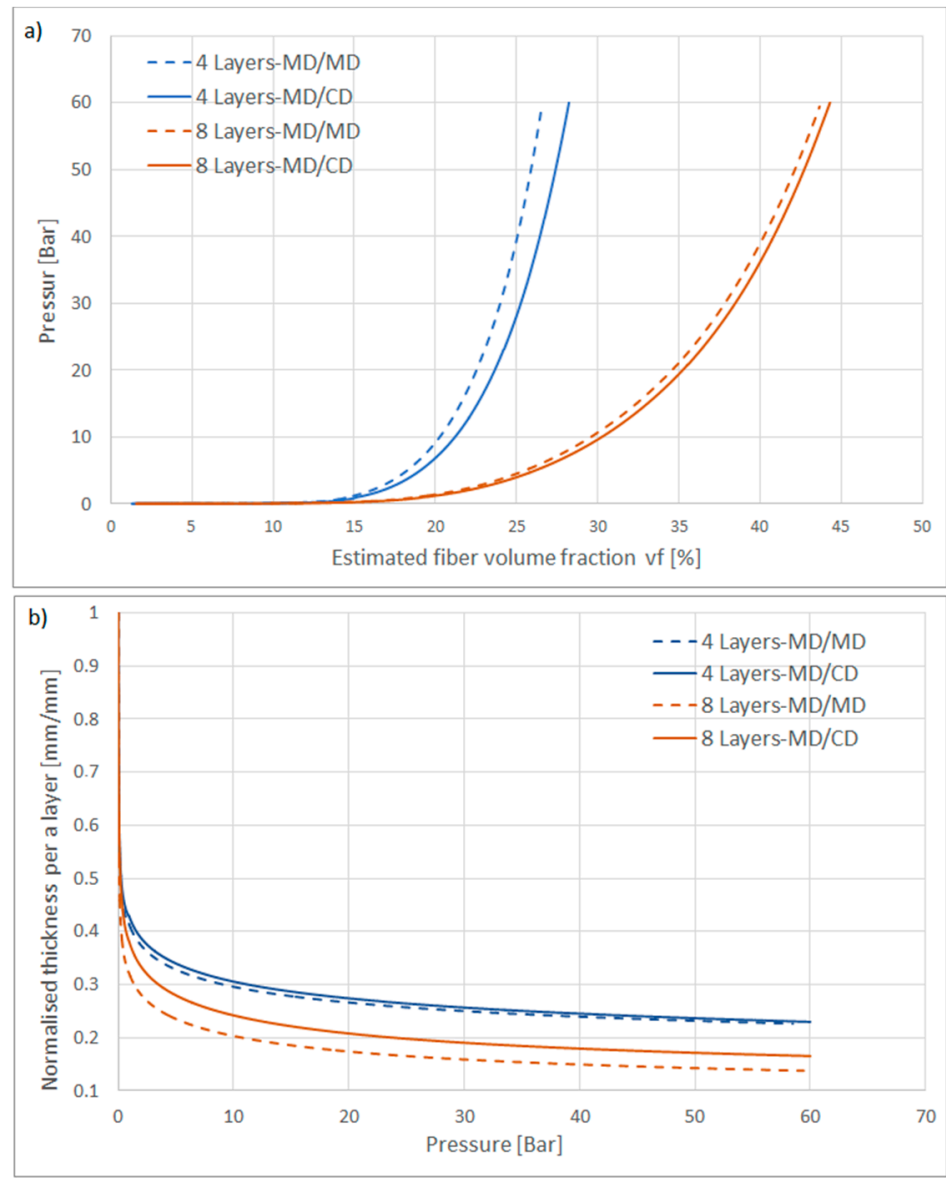

Figure 7. Pressure-estimated fibre volume fraction curve of 4 and 8 layers of nonwoven material tested in MD/MD and MD/CD laminate sequence (a) and normalised thickness of preform per layer of tested samples relative to its thickness per layer at 0.01 bar as a function of applied pressure (b).

\subsubsection{Tensile and Bending Behaviours of rCF Nonwoven Material}

The uniaxial tensile test is conducted on the nonwoven material in three directions $\left(\mathrm{MD}, \mathrm{CD}\right.$, and in bias $\left(45^{\circ}\right)$ direction). The normalised tensile load relative to the specimen 
width is given in accordance with the applied strain in Figure 8a for the three directions. The presented curves are the means of three samples in each direction. The maximum executed normalised force and longitudinal strain at this force are reported in Table 1. Low resistance is observed, whereas a high deformation is demonstrated before complete failure, as shown in $[7,33]$. The nonwoven material in the $\mathrm{CD}$ direction showed superior maximum force. However, it shows less failure strain that was two times lower than the nonwoven performance in $\mathrm{MD}$ and $45^{\circ}$ directions. The nonwoven shows similar tensile behaviour in both $\mathrm{MD}$ and $45^{\circ}$ directions in terms of rigidity, maximum load, and failure strain.

(a)

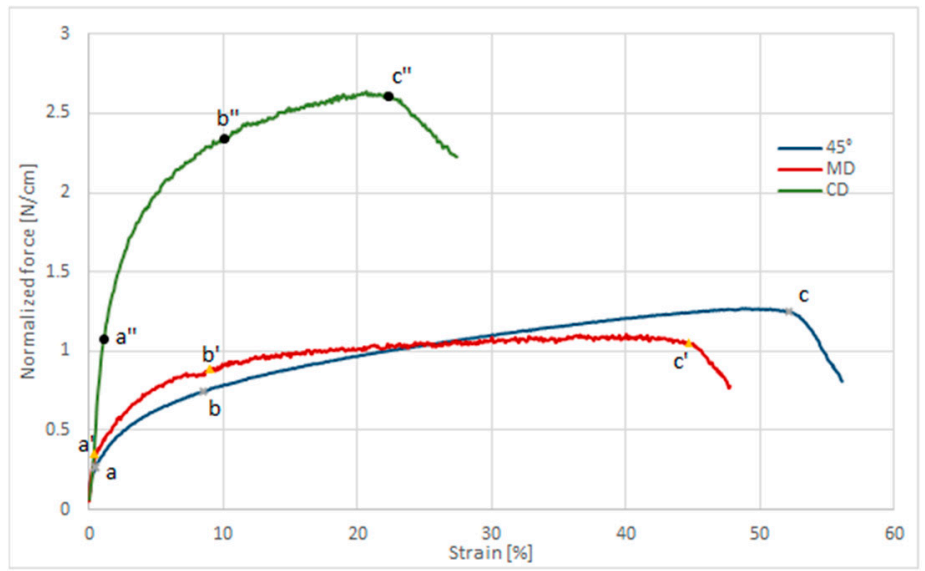

(b)

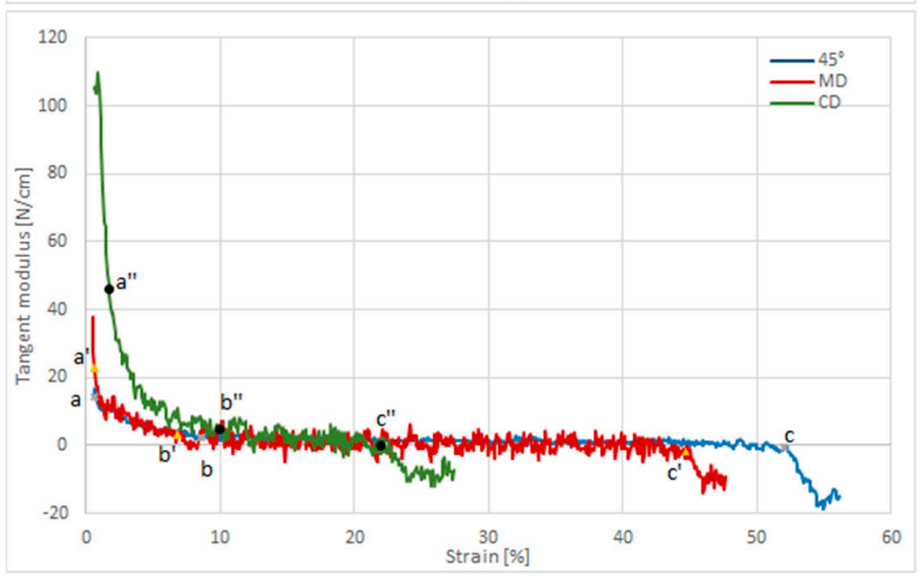

Figure 8. Normalised force-strain curve issued from tensile test of dry nonwoven material executed in three directions $\left(45^{\circ}, \mathrm{MD}, \mathrm{CD}\right)(\mathbf{a})$, and tangent modulus of unidirectional extension in the three directions (b).

Table 1. Mean values of uniaxial tensile properties and flexural rigidity of the nonwoven in three direction $\mathrm{MD}, \mathrm{CD}$, and $45^{\circ}$; standard deviations are given in brackets.

\begin{tabular}{ccccc}
\hline & & 45 & MD & CD \\
\hline \multirow{2}{*}{ Uniaxial tensile } & Maximum normalized force $[\mathrm{N} / \mathrm{cm}]$ & $6.31(0.53)$ & $5.36(0.68)$ & $13.06(0.26)$ \\
& Strain at maximum force $[\%]$ & $52.72(1.44)$ & $46.54(1.75)$ & $22.71(2.47)$ \\
\hline Flexural rigidity & Rigidity per unit width $[\mathrm{N} . \mathrm{m} / \mathrm{cm}]$ & $104(3.7)$ & $101(7.6)$ & $221(11.8)$ \\
\hline
\end{tabular}

Three characterising phases are distinguished for the normalised force-strain curves for the three nonwoven materials, as in Figure 8a, associated with distinguished phases on the tangent tensile modulus-strain curves in Figure $8 \mathrm{~b}$. At first, the nonwoven material shows a significant resistance due to the individual fibres stretching, which is transmitted between fibres by friction. The fibres' contribution at this initial resistance depends on their orientation in the nonwoven sheet relative to the loading direction. This can be observed by 
comparing the difference in initial rigidity between the three nonwoven materials regarding their orientation on the tensile machine, as in Figure 8b. The specimen extended at the CD orientation shows a higher initial rigidity in comparison with other samples extended at the MD and $45^{\circ}$ orientation. This can be explained by the preferred fibre orientation in the nonwoven material and the $\mathrm{CD}$ direction, corresponding to the production direction on the card machine, as explained in Section 2. This preferred fibre orientation was diagnosed by the FFT image analysis technique with a degree of anisotropy of 0.4, as shown in Figure 6. The initial rigidity is considerably degraded, with a stain increase in a linear manner due to the fibre decrimping. At this stage, no local tearing or damage was observed in the specimen plan. The contribution of fibre stiffness is more important in the CD sample as the amount of fibre aligned in the loading direction is more important in comparison with the MD and $45^{\circ}$ specimen. Therefore, the force magnitude at the end of this phase, characterised by altering the nonwoven behaviour to a nonlinear behaviour (point-a" in Figure 8), is higher for CD specimens in comparison with MD and $45^{\circ}$ specimens (pointa and $a^{\prime}$ in Figure 8). By considering the tangent modulus at the end of this first part (point-a' and a") in the MD specimen compared with the CD one to estimate the anisotropy level in tensile behaviour, a ratio of 0.47 is obtained. The normalised force-strain curve maintains a non-linear behaviour for three orientations with a degradation in the tangent modulus until conversion into a linear behaviour at point- $b, b^{\prime}$, and $b^{\prime \prime}$, Figure 8 . This nonlinear behaviour can be attributed to the governing local fibre rotation and sliding, as the transmitted efforts between fibres exceed the inter-fibres' tangential cohesion stress (static friction limit). In this phase, damage initiation is expected in the weakest region of the specimen (less inter-fibre cohesion) in the form of a local tear. In the next phase (b-c, Figure 8) the tear increases with the fibre rotation and sliding associated with the fibres bending, resulting in a linear behaviour with too-low constant rigidity. This phase ends with a fibre separation associated with a drop tensile load. In the CD specimen, as a higher quantity of fibre is already oriented in the load direction, the fibre rotation is less expected on the $b^{\prime \prime}-c^{\prime \prime}$ phase for this specimen, which results in a lower strain value at the end of this phase in comparison with other orientations.

The flexural rigidity of the nonwoven is evaluated in three directions $(M D, C D$, and $45^{\circ}$ ). The obtained values are given in Table 1 . Similarly to the tensile rigidity at the end of the first phase of linear behaviour (point-a, a', and a" on Figure 8), a ratio of 0.456 is obtained between the flexural rigidity in MD direction relative to that in the CD direction. This coincides with the estimated anisotropy index obtained by the image analysis. This highlights the dependency of the mechanical properties on the fibre orientation in the nonwoven material. In the $45^{\circ}$ tested specimen, a non-significant difference is observed in the flexural rigidity in this direction, relative to the MD direction. The same observation is noticed in the tensile test. This is because a similar number of fibres is oriented in the testing direction.

\subsubsection{Preforming Behaviour of rCF Nonwoven Materials}

The preforming behaviour of the rCF nonwoven material is analysed in the three orientations $\left(0^{\circ}, 45^{\circ}\right.$, and $\left.90^{\circ}\right)$. The orientation of the nonwoven samples after preforming is given from captured top-view images in Figure 9. The specimen contour is tracked for each specimen after preforming, draw-in distances, and orientations are analysed. The bold red arrows sketched on the edges of the sample, as in Figure 9a-c, show the orientation of the displacement vector of a point on the edge of the sample in the deformed state relative to its initial position. For the sample placed at $0^{\circ}$ (MD-axis along the $x$-axis), shown in Figure $9 \mathrm{a}$, the displacement in the $y$-direction is the same as that in the CD direction of the nonwoven. For the sample at $45^{\circ}$ (MD and CD along the diagonal), Figure $9 \mathrm{~b}$, this displacement mainly occurs along the sample diagonal. For the sample at $90^{\circ}$ (MD-axis along $y$-axis), Figure 9c, this displacement also mainly occurs in the CD direction. The maximum draw-in always occurs along the CD direction of the nonwoven, independently of the sample orientation. This direction, for the nonwoven, led to higher inter-fibre cohesion in the uniaxial tensile 
test, as shown in Figure 8. The sample is driven by punch movement to conform the punch 3D shape from the compaction/friction zone between the two plates: die and blank holder. Thus, the sample slips on the plate with an axisymmetric in-plane tension, as described for woven fabrics in $[34,35,38-40]$. To evaluate deformation and the appearance of tears in the nonwoven structure, the direction of local stretching on the specimens is estimated. This is achieved by evaluating the transformation of concentric circles marked in the initial state before preforming Figures $3 \mathrm{c}$ and $9 \mathrm{I}$ into ellipses, and examining the inter-gape change between them. As shown in Figure $9 \mathrm{a}-\mathrm{c}$, the initial circles are deformed into ellipses, which mean that the deformation is not identical along the two main axes. The nonwoven is stretched in one direction more than in the other. For the nonwoven samples tested in three orientations $\left(0^{\circ}, 45^{\circ}\right.$, and $\left.90^{\circ}\right)$, the samples are always more stretched along the manufacturing direction, $\mathrm{MD}$, in comparison with the orthogonal direction, $\mathrm{CD}$, as seen in Figure 9a-c. To quantify the observed transformation of the circle into an ellipse, the eccentricity of the deformed ellipse is estimated by determining the ellipse main axis $(\vec{a})$ orientation relative to the nonwoven main direction $(\overrightarrow{M D})$, as in Table 2. The specimens, independently of their orientation on the machine, demonstrate a higher deformation in the MD direction than the $\mathrm{CD}$ direction. This unbalanced deformation is relevant to the eccentricity index, which reaches 0.5 . It can be concluded that the nonwoven material behaviour during preforming is highly associated with the fibre orientation distribution.

।)

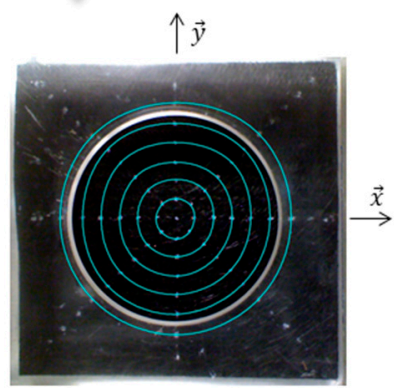

b)

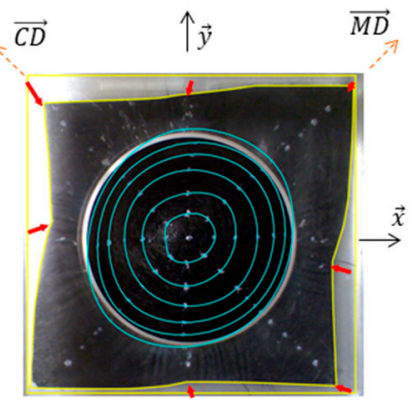

a)

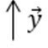

$\overrightarrow{C D}$

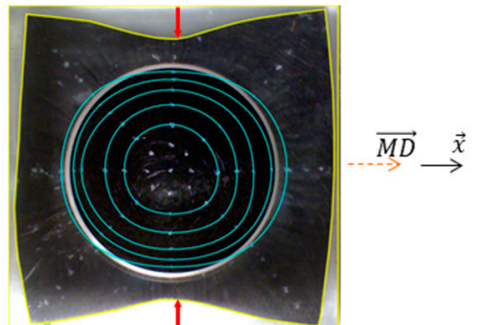

c)

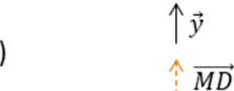

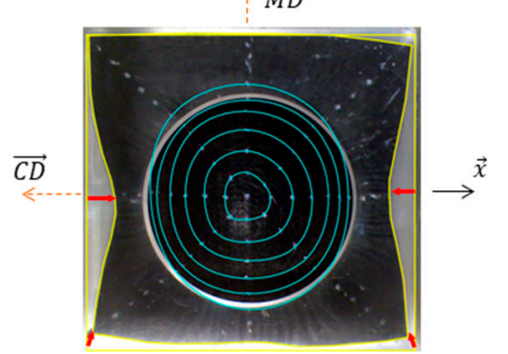

Figure 9. Initial state (I) and preformed nonwoven specimens at $0^{\circ}, 45^{\circ}$, and $90^{\circ}$ angles for nonwoven material MD-axis relative to the machine $x$-axis ( $\mathbf{a}-\mathbf{c}$, respectively). Red bold arrows indicate the maximum draw-in.

Table 2. Characteristics of the deformed ellipse on preformed samples.

\begin{tabular}{cccc}
\hline Specimen Orientation & $\mathbf{0}^{\circ}$ & $\mathbf{4 5}$ & $\mathbf{9 0}^{\circ}$ \\
\hline Eccentricity index & 0.52 & 0.48 & 0.57 \\
\hline Main axis orientation $(\vec{a}, \overrightarrow{M D})$ & $0^{\circ}$ & $0^{\circ}$ & $0^{\circ}$ \\
\hline
\end{tabular}




\subsection{Composite}

Two composite laminates are manufactured from four layers of recycled nonwoven carbon fibre stacked in the same orientation with polypropylene resin. A uniaxial tensile test is performed on the first laminate in the manufacturing direction (MD) of the nonwoven and in the cross direction (CD) on the second laminate. The stress-strain curve of the tensile test is shown in Figure 10. The average stress is considered at each strain value on the presented curve, with STDV added at an interval of 40 recorded data points (this interval is adapted to maintain the visibility of the curve). The fibre and void volume fraction are estimated in the two manufactured laminates based on the material density and are given in Table 3. The longitudinal tensile modulus, tensile strength, and failure strain from the uniaxial tensile test are listed in Table 3. The composite samples show a slightly higher strain at rupture in the MD direction than the $\mathrm{CD}$ direction. However, a significant difference is captured in the tensile modulus and strength between the two directions. A ratio of 0.5 is reported for the tensile modulus in the MD direction relative to that in the $\mathrm{CD}$ direction and, similarly, a ratio of 0.46 is obtained for the tensile strength. In correlation with the anisotropy index obtained by the image analysis, the tensile modulus and strength seem to be highly impacted by the anisotropy that issued from the fibre orientation distribution. However, no significant impact was observed on the failure strain in the two directions. The anisotropy in the fibre orientation distribution in the nonwoven structure, estimated as 0.4 by means of image analysis, has a significant impact on the tensile behaviour and flexural rigidity of the dry nonwoven preform, and the same impact is reported at the composite scale regrading tensile modulus and strength, as in Figure 11.

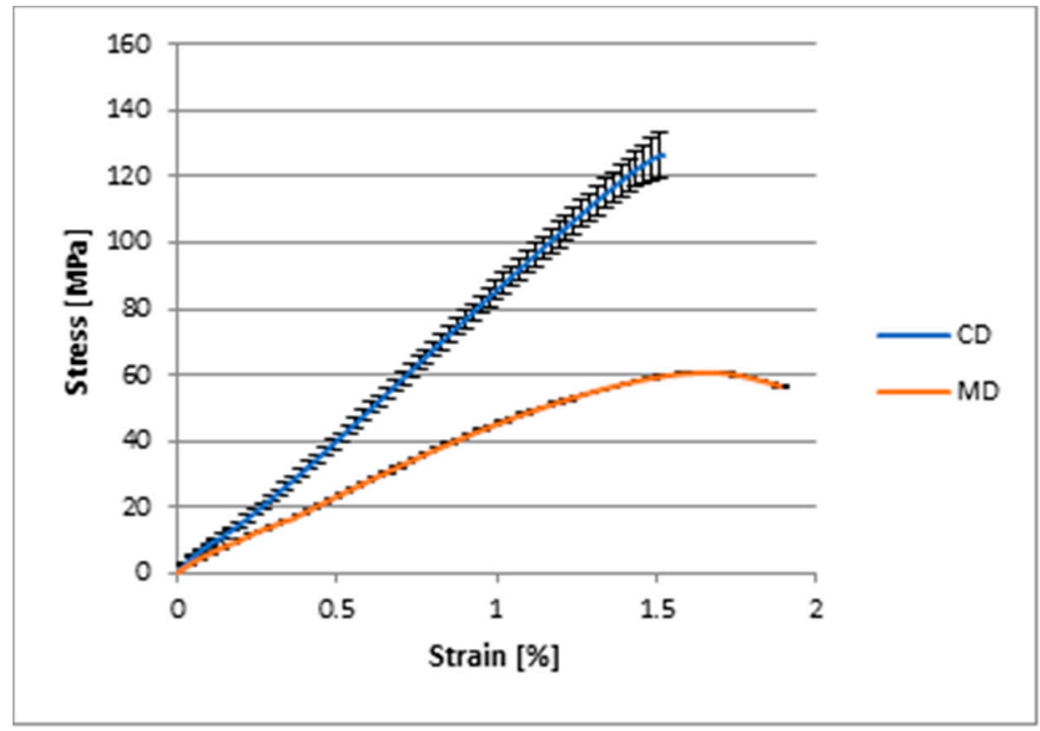

Figure 10. Stress-strain curve of tensile test performed on composite samples in both CD and MD directions.

Table 3. Tensile properties of manufactured composite from the nonwoven recycled carbon fibre.

\begin{tabular}{ccccccc}
\hline Orientation & Thickness $(\mathbf{m m})$ & $\boldsymbol{v}_{\boldsymbol{f}} \mathbf{( \% )}$ & $\boldsymbol{v}_{\boldsymbol{p}} \mathbf{( \% )}$ & $\begin{array}{c}\text { Tensile Modulus } \\
\mathbf{( G P a )}\end{array}$ & $\begin{array}{c}\text { Tensile Strength } \\
\mathbf{( M P a})\end{array}$ & $\begin{array}{c}\text { Failure Strain } \\
\mathbf{( \% )}\end{array}$ \\
\hline $\mathrm{MD}$ & $2.25(0.03)$ & $18.9(0.97)$ & $3.2(2.2)$ & $4.42(0.13)$ & $61.86(1.74)$ & $1.78(0.2)$ \\
\hline $\mathrm{CD}$ & $2.29(0.06)$ & $19.4(0.54)$ & $4.6(0.9)$ & $8.83(0.71)$ & $132.2(13.07)$ & $1.63(0.37)$ \\
\hline
\end{tabular}




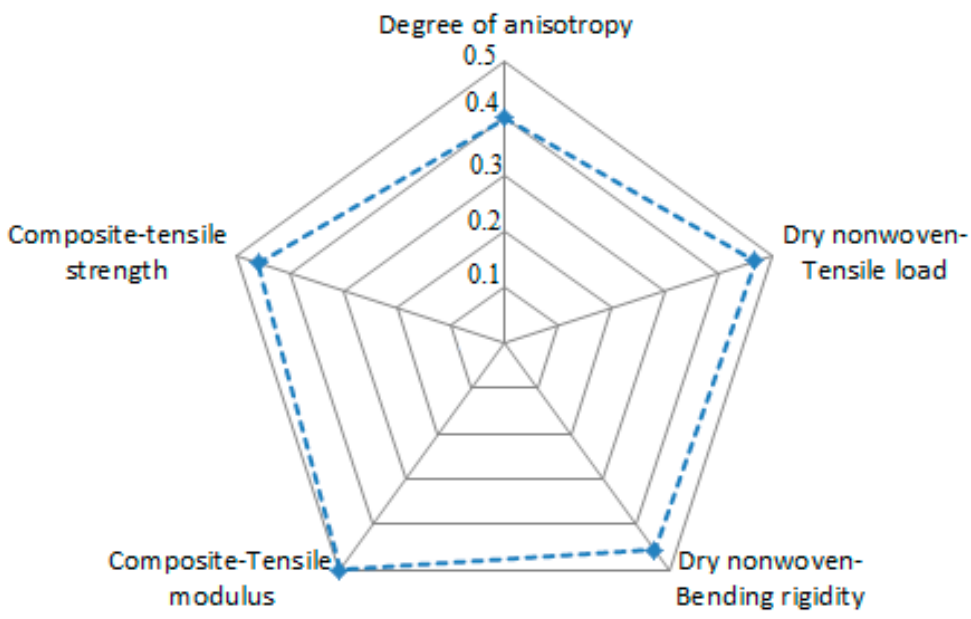

Figure 11. Anisotropy index and ratios characterising parameters between the two main directions (MD and $\mathrm{CD}$ ) of the nonwoven material.

\section{Conclusions}

Nonwoven preform material presents a promising commercial product to revalorise the recycled carbon fibre and give it a second life in the composite application domain. Therefore, understanding the effect that the material properties and process parameters have on the mechanical performance of the nonwoven at each scale, in addition to understanding the interaction between the different scales, is important to control the physical and mechanical properties of the manufactured composite. The present paper aimed to evaluate the physical properties and mechanical performance of a nonwoven manufactured by the ELG Company from recycled carbon fibre. A fibre orientation distribution analysis was conducted of captured SEM micrographs, and anisotropy was revealed in the fibre distribution. A preferred orientation was noticed for the fibre in the cross direction, $\mathrm{CD}$, of the nonwoven. This structural anisotropy causes a significant anisotropy in the uniaxial tensile behaviour and flexural rigidity in the dry nonwoven preform material. Further, anisotropy is observed in the same index order in the mechanical properties of the manufactured composite. Future research should be conducted to track the effect of the nonwoven anisotropy in fibre orientation on the flexural and inter-laminar behaviour of the composite, considering the effect of the layer number and layer orientation sequence.

The recycled nonwoven carbon fibre demonstrated multi-phase behaviour under a uniaxial load, with low resistance and a high dependency on the fibre orientation distribution. The nonwoven in the cross direction (CD) showed higher resistance to tensile load, before the structural rigidity declined in comparison with other directions (MD and $45^{\circ}$ ). In the last two directions, a similar behaviour was observed, and the nonwoven structure showed large deformation before the total rupture, while in the CD direction, the nonwoven has less strain at rupture. Contrary to the first phase, governed by the fibre's high rigidity, this phase of long deformation with a too-low rigidity in the nonwoven behaviour is governed by the inter-fibre rotation and local sliding. The passage between phases is attributed to the initiation of inter-fibre sliding, as well as a rotation, accompanied by the appearance and growth of tears. Thus, the structural behaviour of the nonwoven material is influenced by the fibre orientation distribution and mainly controlled by the inter-fibre cohesion force. This cohesion force essentially depends on the inter-fibre contact surface and interaction properties (coefficients defining friction between fibres in contact). Future research should be conducted to understand the relationship between fibre interaction and the performance of the nonwoven material. Furthermore, it would be interesting to determine the effects that the fibre geometry and tensile properties have on the interaction between fibres, in addition to understanding the influence of the nonwoven manufacturing process parameters on this fibre interaction, especially for recycled carbon fibre. Therefore, 
the geometrical and tensile properties of the used fibre are reported here to contribute to understanding nonwoven behaviour investigated in future research.

The behaviour of the nonwoven material is also investigated under compaction load. A high influence on the number of the stacked layers is noticed when the test is conducted with four and eight layers. The orientation of the stacked layer has a significant impact with a lower number of layers. The layers are more compacted when the orientation of successive layers differs between two orthogonal orientations (CD/MD) in comparison with the same orientation (MD/MD). However, the impact of the layer number is much more important for the compaction behaviour of the nonwoven than the layer orientation sequence.

The performance of the nonwoven when preforming is also related to its anisotropy. A higher draw-in distance always occurred in the preferred fibre orientation distribution, while a lower distance was obtained in the orthogonal direction. Over a long direction with less draw-in distance, tears are expected.

Author Contributions: Conceptualization, A.R.L. and D.S.; methodology, J.I.; validation, A.R.L. and D.S.; Investigation: J.I.; writing—original draft preparation: A.R.L. and D.S.; supervision: D.S.; writing-review and editing: A.R.L. and D.S. All authors have read and agreed to the published version of the manuscript.

Funding: This research received no external funding.

Institutional Review Board Statement: Not applicable.

Informed Consent Statement: Not applicable.

Data Availability Statement: Not applicable.

Conflicts of Interest: The authors declare no conflict of interest.

\section{References}

1. Sauer, M.; Kuhnel, M. Composites Market Report 2019-Market Developments, Trends, Outlook and Challenges. Carbon Compos. 2019. Available online: https://composites-united.com/media/3988/eng_ccev_market-report_2019_short-version.pdf (accessed on 30 November 2021).

2. Das, S.; Warren, J.; West, D.; Schexnayder, S.M. Global Carbon Fiber Composites Supply Chain Competitiveness Analysis; Technical Report of CEMACS; Oak Ridge National Laboratory; The University of Tennessee: Knoxville, TN, USA, 2016.

3. Snudden, J.; Ward, C.; Potter, K. Reusing automotive composites production waste. Reinf. Plast. 2014, 58, 20-27. [CrossRef]

4. Hagnell, M.; Åkermo, M. The economic and mechanical potential of closed loop material usage and recycling of fibre-reinforced composite materials. J. Clean. Prod. 2019, 223, 957-968. [CrossRef]

5. Asmatulu, E.; Twomey, J.M.; Overcash, M. Recycling of fiber-reinforced composites and direct structural composite recycling concept. J. Compos. Mater. 2014, 48, 593-608. [CrossRef]

6. Khurshid, M.F.; Hengstermann, M.; Hasan, M.M.B.; Abdkader, A.; Cherif, C. Recent developments in the processing of waste carbon fibre for thermoplastic composites-A review. J. Compos. Mater. 2020, 54, 1925-1944. [CrossRef]

7. Barnett, P.R.; Gilbert, C.L.; Penumadu, D. Repurposed/recycled discontinuous carbon fiber organosheet development and composite properties. Compos. Part C Open Access 2021, 4, 100092. [CrossRef]

8. Manis, F.; Stegschuster, G.; Wölling, J.; Schlichter, S. Influences on Textile and Mechanical Properties of Recycled Carbon Fiber Nonwovens Produced by Carding. J. Compos. Sci. 2021, 5, 209. [CrossRef]

9. Barnett, P.R.; Ghossein, H.K. A Review of Recent Developments in Composites Made of Recycled Carbon Fiber Textiles. Textiles 2021, 1, 433-465. [CrossRef]

10. Akonda, M.; Stefanova, M.; Potluri, P.; Shah, D. Mechanical properties of recycled carbon fibre/polyester thermoplastic tape composites. J. Compos. Mater. 2017, 51, 2655-2663. [CrossRef]

11. Hengstermann, M.; Raithel, N.; Abdkader, A.; Hasan, M.; Cherif, C. Development of new hybrid yarn construction from recycled carbon fibers for high performance composites. Part-I: Basic processing of hybrid carbon fiber/polyamide 6 yarn spinning from virgin carbon fiber staple fibers. Text. Res. J. 2016, 86, 1307-1317. [CrossRef]

12. Wei, H.; Nagatsuka, W.; Lee, H.; Ohsawa, I.; Sumimoto, K.; Wan, Y.; Takahashi, J. Mechanical properties of carbon fiber paper reinforced thermoplastics using mixed discontinuous recycled carbon fibers. Adv. Compos. Mater. 2017, 27, 19-34. [CrossRef]

13. Lee, H.; Choi, C.-W.; Jin, J.-W.; Huh, M.; Lee, S.; Kang, K.-W. Homogenization-based multiscale analysis for equivalent mechanical properties of nonwoven carbon-fiber fabric composites. J. Mech. Sci. Technol. 2019, 33, 4761-4770. [CrossRef]

14. Tunák, M.; Antoch, J. Monitoring homogeneity of textile fiber orientation. Text. Res. J. 2018, 88, 1226-1243. [CrossRef]

15. Gager, V.; Legland, D.; Bourmaud, A.; Le Duigou, A.; Pierre, F.; Behlouli, K.; Baley, C. Oriented granulometry to quantify fibre orientation distributions in synthetic and plant fibre composite preforms. Ind. Crop. Prod. 2020, 152, 112548. [CrossRef] 
16. Yu, B.; Zhao, X.; Zeng, Y.; Qi, D. The influence of process parameters on needle punched nonwovens investigated using image analysis. RSC Adv. 2017, 7, 5183-5188. [CrossRef]

17. Khurshid, M.F.; Abdkader, A.; Cherif, C. Processing of waste carbon and polyamide fibres for high-performance thermoplastic composites: Influence of carding parameters on fibre orientation, fibre length and sliver cohesion force. J. Text. Inst. 2020, 111, 1277-1287. [CrossRef]

18. Jlassi, S.; Berthet, F.; Bernhart, G. Investigation of mechanical properties of nonwoven second generation composite material elaborated through a mixture of carbon fibers and filament lengths. In Proceedings of the ECCM18-18th European Conference on Composite Materials, Athens, Greece, 24-28 June 2018.

19. AFNOR. NF EN 12127-Textiles-Fabrics-Determination of Mass Per Unit Area Using Small Samples; AFNOR: Dartford, UK, 1998.

20. AFNOR. NF EN ISO 5084-Textiles-Determination of Thickness of Textiles and Textile Products; AFNOR: Dartford, UK, 1996.

21. Schneider, C.A.; Rasband, W.S.; Eliceiri, K.W. NIH Image to ImageJ: 25 years of image analysis. Nat. Methods 2012, 9, 671-675. [CrossRef]

22. Barocas, V. Image-Based Fiber Orientation and Alignment Calculator, University of Minnesota. Available online: https: / license. umn.edu/product/image-based-fiber-orientation-and-alignment-calculator (accessed on 11 November 2021).

23. Syerko, E.; Oter, L.; Pawar, A.; Binetruy, C.; Advani, S.G.; Eck, B. Comparative Study of Methods for the Quantification of Fiber Orientations of Composite Reinforcements. In Proceedings of the 21ème Journées Nationales des Composites, Bordeaux, France, 1-3 July 2019.

24. Sander, E.A.; Barocas, V.H. Comparison of 2D fiber network orientation measurement methods. J. Biomed. Mater. Res. Part A 2009, 88A, 322-331. [CrossRef]

25. Ivanov, D.S.; van Gestel, C.; Lomov, S.V.; Verpoest, I. Local compressibility of draped woven fabrics. In Proceedings of the ECCM15-15th European Conference on Composite Materials, Venice, Italy, 24-28 June 2012.

26. Nguyen, Q.; Vidal-Sallé, E.; Boisse, P.; Park, C.H.; Saouab, A.; Bréard, J.; Hivet, G. Mesoscopic scale analyses of textile composite reinforcement compaction. Compos. Part B Eng. 2013, 44, 231-241. [CrossRef]

27. Yousaf, Z.; Potluri, P.; Withers, P. Influence of Tow Architecture on Compaction and Nesting in Textile Preforms. Appl. Compos. Mater. 2017, 24, 337-350. [CrossRef]

28. Yong, A.; Aktas, A.; May, D.; Endruweit, A.; Lomov, S.; Advani, S.; Hubert, P.; Abaimov, S.; Abliz, D.; Akhatov, I.; et al. Experimental characterisation of textile compaction response: A benchmark exercise. Compos. Part A Appl. Sci. Manuf. 2021, 142, 106243. [CrossRef]

29. Danzi, M.; Schneeberger, C.; Ermanni, P. A model for the time-dependent compaction response of woven fiber textiles. Compos. Part A Appl. Sci. Manuf. 2018, 105, 180-188. [CrossRef]

30. Corbin, A.-C.; Soulat, D.; Ferreira, M.; Labanieh, A.-R.; Gabrion, X.; Malécot, P.; Placet, V. Towards hemp fabrics for highperformance composites: Influence of weave pattern and features. Compos. Part B Eng. 2019, 181, 107582. [CrossRef]

31. AFNOR. NF EN ISO 9073-3-Textiles-Test Method for Nonwoven-Part 3: Determination of Tensile Strength and Elongation; AFNOR: Dartford, UK, 1992.

32. AFNOR. NF EN ISO 9073-7-Textiles-Test Method for Nonwoven-Part 7: Determination of Bending Length; AFNOR: Dartford, UK, 1998.

33. Omrani, F.; Wang, P.; Soulat, D.; Ferreira, M.; Ouagne, P. Analysis of the deformability of flax-fibre nonwoven fabrics during manufacturing. Compos. Part B Eng. 2017, 116, 471-485. [CrossRef]

34. Dufour, C.; Boussu, F.; Wang, P.; Soulat, D. Local strain measurements of yarns inside of 3D warp interlock fabric during forming process. Int. J. Mater. Form. 2018, 11, 775-788. [CrossRef]

35. Corbin, A.-C.; Soulat, D.; Ferreira, M.; Labanieh, A.-R. Influence of Process Parameters on Properties of Hemp Woven Reinforcements for Composite Applications: Mechanical Properties, Bias-extension Tests and Fabric Forming. J. Nat. Fibers 2020, 17, 1-13. [CrossRef]

36. Berges, M.; Léger, R.; Placet, V.; Person, V.; Corn, S.; Gabrion, X.; Rousseau, J.; Ramasso, E.; Ienny, P.; Fontaine, S. Influence of moisture uptake on the static, cyclic and dynamic behaviour of unidirectional flax fibre-reinforced epoxy laminates. Compos. Part A Appl. Sci. Manuf. 2016, 88, 165-177. [CrossRef]

37. ASTM International. ASTM D3039/D3039M-17-Standard Test Method for Tensile Properties of Polymer Matrix Composite Materials; ASTM International: West Conshohocken, PA, USA, 2017.

38. Gatouillat, S.; Bareggi, A.; Vidal-Sallé, E.; Boisse, P. Meso modelling for composite preform shaping simulation of the loss of cohesion of the woven fibre network. Compos. Part A 2013, 54, 135-144. [CrossRef]

39. Boisse, P.; Hamila, N.; Madeo, A. Analysis of defect developments in composite forming. In The Structural Integrity of Carbon Fiber Composites; Beaumont, P.W.R., Soutis, C., Eds.; Springer International Publishing: New York, NY, USA, 2017.

40. Labanieh, A.R.; Garnier, C.; Ouagne, P.; Dalverny, O.; Soulat, D. Intra-ply yarn sliding defect in hemisphere preforming of a woven preform. Compos. Part A 2018, 107, 432-446. [CrossRef] 